\title{
Grapevine adaptation to drought: New candidate genes for the genotype-dependent response
}

\author{
M.F. Cardone ${ }^{1}$, R. Perniola ${ }^{1}$, C.R. Catacchio ${ }^{2}$, F. Alagna $^{3}$, S. Rotunno ${ }^{1}$, P. Crupi ${ }^{1}$, D. Antonacci ${ }^{1}$, R. Velasco ${ }^{1}$, \\ M. Ventura ${ }^{2}$, and C. Bergamini ${ }^{1}$ \\ ${ }^{1}$ Consiglio per la ricerca in agricoltura e l'analisi dell'economia agraria - Centro di ricerca Viticoltura ed Enologia (CREA- VE), \\ Via Casamassima 148, 70010 Turi (Ba), Italy \\ ${ }^{2}$ Università degli Studi di Bari "Aldo Moro" - Dipartimento di Biologia, via Orabona, 4, 70126 Bari, Italy \\ ${ }^{3}$ ENEA, Agenzia nazionale per le nuove tecnologie, l'energia e lo sviluppo economico sostenibile, Centro Ricerche Trisaia, \\ Rotondella (MT), Italy
}

\begin{abstract}
Grapevine requires irrigation supply for its cultivation, especially in the arid and semiarid geographic areas. As consequence of the severe climatic changes, water consumption is becoming more and more important as environmental and cost factor that needs to be reduced. Water deficiency can affect berry and wine quality depending on the extent of plant perceived stress, which is a cultivar specific trait. In a fouryear project, we tested the physiological and molecular responses to water deficiency of two different table grape cultivars, Italia and Autumn Royal, and we highlighted that they differently adapted to drought stress conditions. Physiological analyses on field-growth plants showed cultivar-specific variations in photosynthetic carbon assimilation and, stomatal conductance under water deficiency. We further combined "omic" analyses to identify candidate genes involved in drought stress response and adaptative traits. Microarray analyses revealed a broad response of cultivar Italia to drought stress conditions characterized by the modulation of 1037 genes involved in biological processes as cell wall organization, carbohydrate metabolism, ROS response, response to hormone and osmotic stress. On the contrary, Autumn Royal response was limited to the modulation of only 29 genes mainly involved in plant stress response, nitrogen metabolism and hormone signal transduction. Our data highlighted that ABA-perception and-signalling are key factors mediating the varietal-specific behavior of the early response to drought.
\end{abstract}

\section{Introduction}

Vine development and grape ripening are sensitive to environmental factors and, among these, water is the most important limiting resource for vineyards grown in in arid and semi-arid regions (i.e. Mediterranean climate), especially those devoted to table grape production. The cycle for table grape production involves the spring, summer and autumn, and is characterized by a high demand for water. This leads to an enormous consumption of freshwater resources. Thus, in order to promote a more sustainable viticulture, a reduction of water use has become essential. This is the challenge driving the recent researches to identify tolerant varieties with a better adaptation to water deficit.

Vitis vinifera $\mathrm{L}$. has been described as relatively tolerant to water deficit, and regulated deficit irrigation has been advantageously used in wine-production as it induces an increase in total phenolic and anthocyanin content in fruits, which indeed influences the "sensory" characteristics and quality of wines [1,2]. However, water deficit (WD) negatively affects important aspects of table grape production, such as yield, berry size, firmness. Thus, irrigation remains fundamental to overcome water limitations. The effects of deficit irrigation are dependent on the climatic characteristic during growing season, soil type, and timing of application, but also on grapevine cultivar and rootstock type which means that the search of those cultivar, rootstock and their combination displaying higher adaptability and resilience to drought is becoming critical.

Inter-varietal differences and a dynamic physiological response to water availability have been described, thus revealing a different adaptation of grapevine varieties to the environmental conditions and a different ability to respond to water stress [3]. At the physiological level, stomata closure, in response to water status declines, is one of the first responses to water deficit, in order to prevent the hydraulic failure [3]. Many scientists described variation in stomata control and proposed a physiological classification of plants as isohydric or anisohydric. Isohydric species can maintain a constant midday leaf water potential ( $\Psi$ leaf), by closing their stomata, regardless of soil water availability; whereas anisohydric species maintain higher stomatal aperture to optimize photosynthetic activity, but their Uleaf significantly declines as soil water deficit increases [3].

However, this framework is still debated considering that grapevine cultivars can exhibit, for instance, both near iso- or anisohydric behaviours depending on the environmental conditions $[4,5]$. In particular, a key role in the determination of the degree of iso/anysohydricity is played by the hydraulic properties of the soil $[6,7]$ and by the rootstock [8]. 
At the molecular level, the phytohormone abscisic acid (ABA) plays a key role in mediating the stomatal responsiveness to WD. Recently, comparative studies have investigated the relationship of transcriptomics, metabolomics and physiology in response to water stress $[5,9]$. However, studies integrating high throughput genomic data for the identification of the genetic traits responsible for the genotypic-specific response of grapevine to water deficit stress are still not available. In the present paper we described results obtained in the project MIUR-PON02_00186_2866121ECO_P4 "Promotion of eco-sustainable processes for the enhancement of Apulian agricultural production" (20112015). During this period, we first investigated the influence of rootstock and irrigation treatments on the vine water status and further we tested the physiological and molecular responses to water deficiency of two different table grape cultivars, Italia and Autumn Royal under WD conditions. This paper summarizes some of the results reported in Perniola et al. 2016 [10] and Catacchio et al. 2019 [11].

Analyses were performed directly on the field in order to evaluate the varietal and rootstock adaptation to growing conditions with reduced irrigation. Comparison of two different rootstocks combined with three seedless cultivars revealed that rootstocks and irrigation levels may play a direct role in grape quality ant thus the choice of the appropriate rootstock might be crucial to improve grape quality under WD. Moreover, transcriptomic assays on apexes under WD and at full irrigation (FI) conditions revealed a great divergence in the response of these two cultivars, thus highlighting genotype-specific responses.

More, by investigating NGS data, we were able to identify genomic variants putatively associated with the different ability to respond to water deficit.

\section{Material and methods}

\subsection{Plant material and irrigation treatments}

We carried out the study in an experimental vineyard of the Agricultural Research Council, Research Centre for Viticulture and Enology in Turi (Apulia Region) in 2013 and 2014. The vineyard was located in a trial site on a hilly area (in Turi, southern Italy, long. $40.57^{\circ} \mathrm{E}$, lat. $17.00^{\circ} \mathrm{N}$ ) at about $190 \mathrm{~m}$ a.s.l. For the analysis of irrigation treatments of rootstocks three seedless varieties (Crimson Seedless- CS, Autumn Royal-AR, and Summer Royal$\mathrm{SR}$, grafted on two rootstocks $140 \mathrm{Ru}$ (V. berlandieri $\times$ $\mathrm{V}$. rupestris) and 34EM (V. berlandieri $\times$ V. riparia) were studied. Further two Vitis vinifera L. cultivars, AR and Italia (It), grafted onto "140 Ru" were used as plant material. For detailed growth conditions and climate see [10].

Two irrigation treatments, based upon a percentage of the net irrigation requirements $[\mathrm{NIR}=\mathrm{ETc}$ (crop evapotranspiration) - Effective rainfall] from fruit set till harvest, were applied: control full irrigation (FI) and water deficit irrigation (WD) at 100 and $60 \%$ of NIR, respectively. ETc was estimated using varying crop coefficients $(\mathrm{kc})(\mathrm{ETc}=\mathrm{ETo} \times \mathrm{kc})$ based on those proposed by FAO and adjusted for the Mediterranean area and ETo values. According to the typical practice adopted in the Apulian region, the vines were drip-irrigated by means of irrigation lines installed $180 \mathrm{~cm}$ above the soil surface with drippers spaced $70 \mathrm{~cm}$ apart and set to supply water at a constant pressure with two $8 \mathrm{~L} \mathrm{~h}-1$ drippers vine -1 . Except for the irrigation treatments, all the other standard cultural practices in the vineyard were applied equally to all vines.

Vine water status and leaf gas exchange were calculated during the steady period of the water potential diurnal curve (generally between 12.30 and $13.30 \mathrm{~h}$ ), following the protocols described in $[10,11]$.

\subsection{Fruit quality analysis}

For each thesis 7-bunch samples of were randomly harvested at commercial maturity (September 8, 2013) according to a sugar-acid ratio $>25$. Twenty berries from each bunch were collected, weighed, and their firmness was measured using a deformation tester (Digital Fruit Firmness Tester, Forlì, Italy). Finally, juice was extracted from each sample and used to measure $\mathrm{pH}$, total soluble solids (TSS) as ${ }^{\circ}$ Brix, and titratable acidity. Moreover, three 10-berry samples from the bunches were randomly collected and frozen at $-20^{\circ} \mathrm{C}$ and used for further polyphenols and anthocyanins profiles as described in Perniola et al. [10] . Differences in the quality parameters between the irrigation treatments (FI vs. WD) of the cultivars were tested through pairwaise Student's t-tests by using STATISTICA 8.0 (StatSoft Inc., Tulxa, OK) package and the statistical tools available in excel.

\subsection{Transcriptomic and genomic analysis}

Microarray analyses were carried out on an Agilent custom array according to the manufacturer's instructions (version 6.9.1, Agilent Technologies). Microarray expression data were processed and analysed using the $\mathrm{R}$ package limma ( $\mathrm{R}$ version 3.1.2, limma version 3.23.2).

Transcripts showing a fold change $\geq 2$ with $p<0.05$ were considered as differentially expressed (DEG). In order to have a comprehensive view on the DEGs belonging to specific pathways of interest, an integrated approach based on multiple annotation methods was used. For detailed protocols see Catacchio et al. [11].

Copy number and single nucleotide variations between $\mathrm{AR}$ and It cultivars were retrieved from data produced by Cardone and co-authors following the already described methods [12] (Sequence Read Archive, ID: SRP009057).

\section{Results and discussion}

In the present work, we described the results obtained in the project ECO_P4. We combined physiological studies with transcriptomics and genomics in order to investigate the different ability grape varieties to respond to water stress also in different scion/rootstock combination and highlighted new clues about the genetic bases of these differences.

Most of the previous literature data are based on experiments under controlled conditions, and they are mostly focused on wine grape varieties. We, instead, tested the effects of a reduced irrigation directly on the field in order to investigate how the mentioned different ability reflects an adaptation of the cultivars to the growing conditions. 


\subsection{Effect of WD on different scion/rootstock combinations}

Since the early 1900s in Italy, in viticulture, the root system of the grapevine is defined by the use of rootstock to which the desired cultivar of grapes is grafted.

Rootstock types vary in root distribution and affect scion responses in vigor, yield, fruit quality and other physiological parameters, especially under different irrigation treatments [13]. For that reason, we investigated the influence of rootstock genotype and irrigation treatments on the vine water status and on the quality of three seedless table grape cultivars, AR, SR, and $\mathrm{CS}$ grafted on two different rootstocks, "140 Ru" and "34 EM".

Vine water status was clearly affected by the irrigation treatment, as shown by the seasonal evolution of $\psi 1$ in the three cultivars. The trend was in agreement with several experiments on grapevine which showed that drying the whole root system where irrigation input was either reduced or withheld completely for specified periods of time resulted in a significant decrease of $\psi l$ [14]. Moreover, it is worth noting that irrespective of rootstock type, "Autumn Royal" vines treated with FI showed $\psi 1$ values firstly between -0.5 and -1.0 (zero water deficit) and then in the range between -1.0 and -1.2 (mild water deficit); while in condition of WD, the $\psi l$ values ranged from moderate deficit, before irrigation, to mild water deficit, after irrigation. Finally, WD treatment showed a progressive decay passing from a condition of mild stress (start of the experiment) to strong water deficit in the last analysis.

Comparison of the different scion/rootstock combinations allowed us to demonstrate that "34 EM" was less adapted to limited water conditions with respect to "140 Ru", qualified as drought tolerant.

The effect of cultivar, rootstock, and water supply on grape quality component was measured (Table 1). We found that berry weight was significantly affected by the interaction between cultivar, water supply and rootstock: the highest values (up to $8.6 \mathrm{~g}$ ) were measured for "Autumn Royal" irrigated at $100 \%$ of NIR (FI) and grafted onto "140 Ru".

Instead, cultivar and water supply, separately, were the only factors influencing the bunch weight. The significant influence of water supply on berry size and bunch weight can be due to the duration of the treatment, since previous investigations on grapes have shown that early season WD were particularly effective in reducing fruit size at harvest rather than late season deficit. It could also have been due to the strong water stress conditions that vineyards experienced in this study, considering that, in general, the magnitude of the reduction in berry size is proportional to severity of water stress [4].

Literature suggests a dependency of food texture on water content and water activity because water acts as a plasticizer in hydrophilic foods; in particular, fruit firmness is known to be responsive to the vine water status and postveraison water deficit treatment accelerates fruit softening. In our experiments we found that the three cultivars grafted onto " $140 \mathrm{Ru}$ " appeared on average more resistant to detachment.

Finally with referment to quality traits, a number of experiments have indicated that different irrigation regimes have a significant effect on grape juice composition, although the literature results are often contradictory mainly because the influence of water deficit on sugar and organic acid content of grapevine berries is strictly dependent on cultivar, climate, and operative conditions [4].

As described in Table 1 our results highlighted that rootstocks and irrigation levels may play a direct role in grape quality in terms of berry chemical composition of table grape under water deficit irrigation. In particular, the rootstock "34 EM" seems more suitable under non-limiting water conditions, while the " $140 \mathrm{Ru}$ " might be the better choice under WD.

\subsection{Effect of WD on physiology and berry quality of different cultivars}

Taken into account the results obtained comparing different scion/rootstock combinations and the better performances of the "140Ru", we further analysed the effect of WD on different cultivar genotype. Plants of the seeded cultivar It grafted of "140Ru" were subjected to the same irrigation treatments as previously described for the seedless cultivars (\$par.3.1) and the results were compared to those obtained for AR/140Ru combination.

Irrigation treatment clearly affected vine water status as shown by the seasonal evolution of $\psi$ leaf also in It as observed for AR. However, noteworthy $\psi$ leaf decreased more rapidly in AR than in It and at the end of the treatment differences in $\psi$ leaf were higher in AR.

Excess of irrigation is often practised for table grape production; therefore, a condition of OI (corresponding to an increment of $50 \%$ of water supply with respect to FI) was also tested for cultivar It. As expected, the $\psi$ leaf showed higher levels than in the FI.

The comparison of the physiological overall data suggested that WD treatment resulted in better intrinsic water use efficiency in AR with respect to It.

We also compared the influence of the applied WD on the productivity and fruit quality at harvest time in It compared to those we found for the seedless cultivar AR. We observed a negative influence of WD also for It on cluster numbers and bunch weight, which resulted in a lower production per vine in both cultivars.

Moreover, we also measured a reduction of berry size. Quality parameters such as sugar content, $\mathrm{Ph}$ and total acidity did not show significant variations, despite a global reduction of the sugar production per vine (Table 2).

\subsection{New clues on the molecular bases of grapevine responses to drought}

The physiological and chemical analysis on different cultivars confirmed the strong influence of the cultivar genotype on the responses to WD. For that reason, we investigated the bases of this genotype-dependent responses at molecular level, and for the first time we combined transcriptomic data with genomics to identify new putative candidate genes responsible for such differences.

In order to study the effect on grapevine gene expression of a reduction of irrigation supply in field condition, leaf apexes of plants of AR and It subjected to FI and WD conditions were collected and used for microarray analyses. We compared differentially 


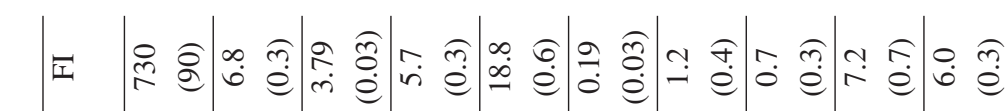

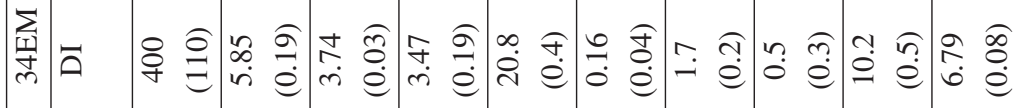

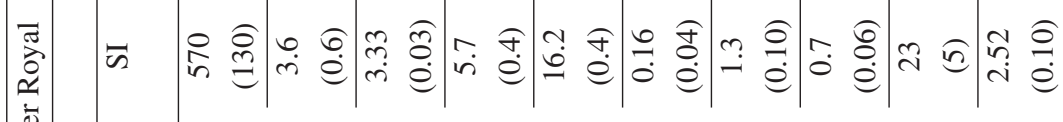

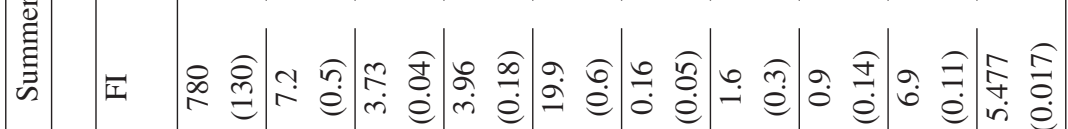
z

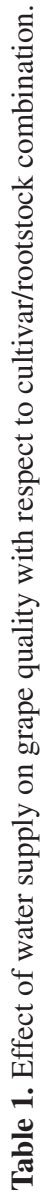

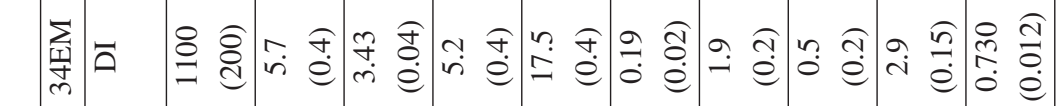

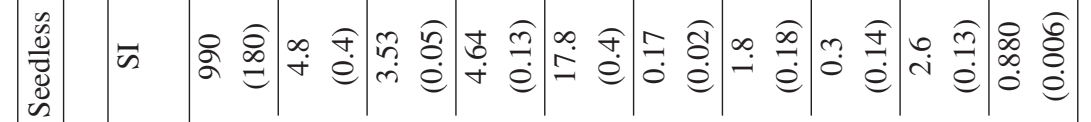

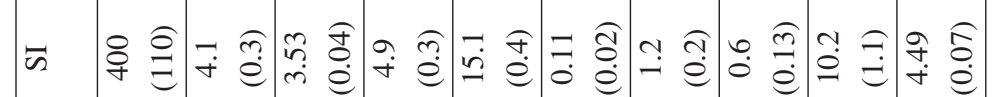

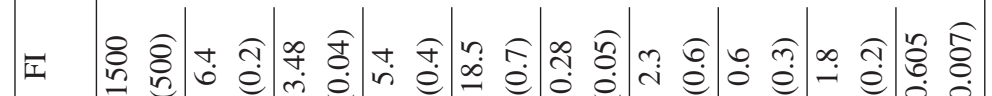
I

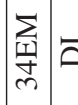

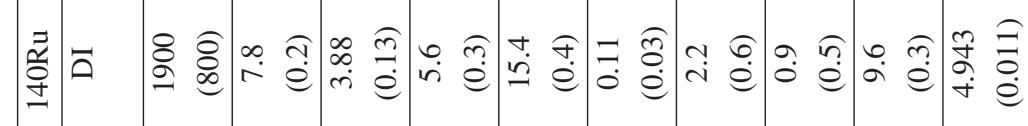

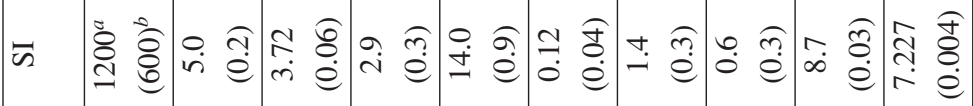

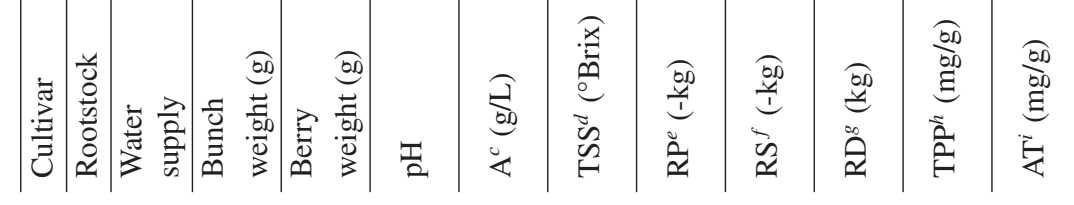

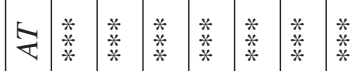

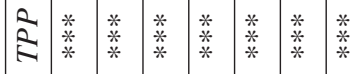

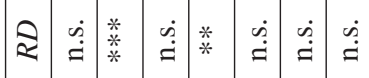

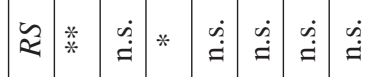

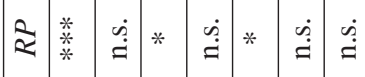

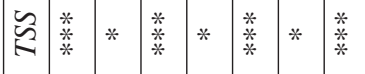

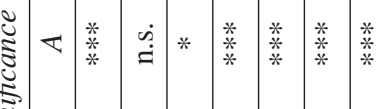

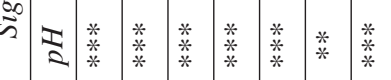

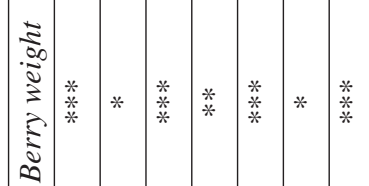

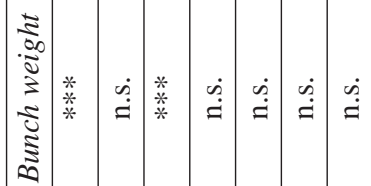

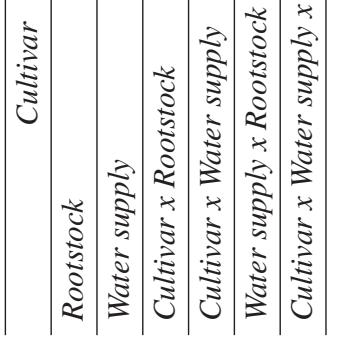


expressed genes (DEGs): (a) in each cultivar under water deficit; (b) between the two cultivars at both FI and WD conditions; (c) between OI and WD in It (Fig. 1).

Networking analyses of the DEGs between FI and WD conditions revealed a cultivar-specific response to WD. In particular, It showed a predominant downregulation of genes involved in the primary metabolism (DNA replication, carbohydrate metabolism, and cell wall organization), coupled with a modulation (up- or down-regulation) of genes involved in the responses to stress (osmotic stress, response to oxygen containing compounds, inorganic substances, and hormones) (Fig. 2).

In contrast, in AR, the modulation of gene expression under WD was limited to a very little number of genes but most of them are specifically involved in the plant response to stress conditions including drought-responsive genes such as desiccation proteins (Fig. 2). To select, among the DEGs between cultivars, those specifically modulated in responses to WD, we intersected them with the DEGs between FI and WD conditions (Fig. 2).

The genes common to these two groups (137 down and 233 up-regulated in AR compared to It) have been used for gene networking analysis (Fig. 2d). The results indicated that $\mathrm{AR}$ at WD showed a predominant up-regulation of genes involved in the following biological processes: cell wall organization, macromolecule metabolic process, amino- and nucleotidesugar metabolism and a predominant down-regulation of genes involved in the response to oxygen containing compounds. These results agree with the modulation of genes belonging to these pathways in It under WD. Interestingly, the analysis indicated nitrogen metabolism as a significant pathway modulated between the two varieties, confirming its importance in determining the different response to WD of the two cultivars.

The strong differences observed between AR and It under WD stress might also depend on a different timing of response between the two cultivars: AR could activate later a more extensive response to WD, similarly to what found for the anisohydric cultivar Sangiovese [5]. This suggests that the genotype-specific responses to WD need to be investigated at the early phases after WD.

According to the important role of phytohormones in the regulation of plant stress response we further focused on DEGs involved in hormone perception and response. We found, respectively, 45 down- and 34 up-regulated genes in response to WD in cultivar It, whereas, four up- and one down-regulated genes were identified in AR. ABA, auxin and ethylene responsive genes were predominant among the DEGs. Three genes characterize the response of AR (up-regulated exclusively in this cultivar) and encode for pathogenesis-related proteins putatively associated to salicylic acid signalling. According to this, we found ABA response elements (ABRE) and ABRE-related motifs in 42 hormoneresponsive DEGs.

As expected, under WD condition, we identified 22 up- and 23 down-regulated genes involved in osmotic and water stress response in It. Among them, six genes encode for dehydration responsive proteins (RD22, RD26, XERICO, DRS1) and transcription factors regulated by ABA (DREB1A, MYB102). Noteworthy, two osmotinlike genes OSM34 generally associated to drought tolerance in other species resulted up- (VIT_02s0025g04340)

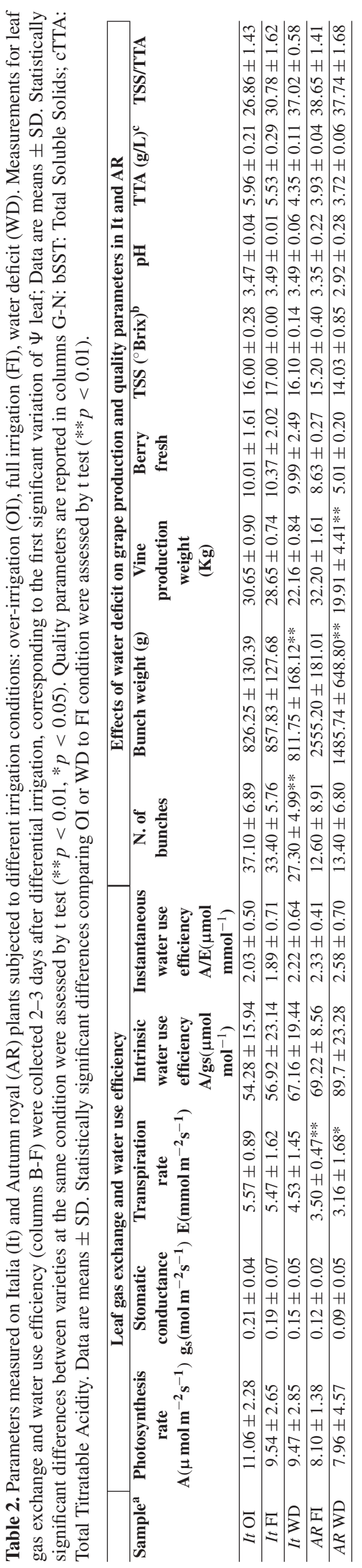




\section{A Regulation under water deficit}

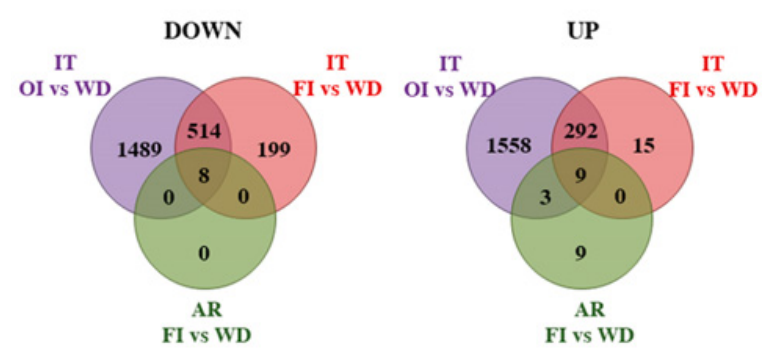

B

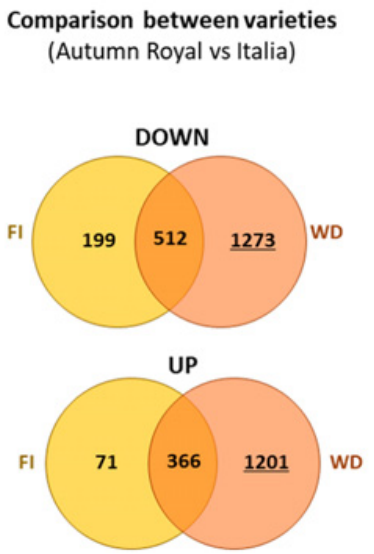

Figure 1. DEGs under WD in Italia and Autumn Royal. As modified by Catacchio et al. (2019). A) Venn diagrams show down- and up-regulated genes between different water conditions. The comparison between OI and FI in It do not reveal any significant differentially expressed gene (DEG). B) Venn diagrams show down- and up-regulated genes between It and AR. The higher variation of gene expression between cultivars is depicted at WD (the number of DEG is underlined). AR: Autumn Royal; IT: Italia; WD: water deficit; FI: full irrigation; OI: over irrigation.

and down-regulated (VIT_02s0025g04230). In contrast to the large number of genes differentially expressed in It, only two genes involved in osmotic stress response resulted differentially expressed in AR, both up-regulated: b-glucanase (VIT_205s0077g01150) and OSM34 (VIT_02s0025g04340), similarly to It.

Modulation of 41 genes involved in the response to oxygen-containing compounds was observed in It. Only one gene involved in these pathways was differentially expressed (up-regulated) in AR: the peroxidase 5-like (RCI3: VIT_14s0060g00520).

In addition, we also found many transcription factors among the DEGs with different modulation in the two cultivars. In fact, we found 61 TF differentially expressed (35 down-regulated, 26 up-regulated) in It versus only one TF differentially expressed (up-regulated) in AR. Most of TFs belong to AP2-EREB, bHLH and MYB families and thus related to the hormone responses.

Overall transcriptomic data were further compared to genomic data derived by NGS data-mining of the two studied cultivars [12], in order to find genotype-specific differences putatively associated to the observed different response to WD. In particular we searched for polymorphic genes between the analysed cultivars involved in abiotic stress. More in detail, we focused our attention on copy number variant $(\mathrm{CNV})$ regions and single nucleotide variants (SNVs) affecting gene transcription. Notably we found that $5 \%$ of the grape genome is variable between the two cultivars. Moreover, among the overall polymorphisms we searched for polymorphic genes involved in stress response or hormone signalling (Table 3 ).

In order to gain insights into the genetic bases of cultivar-specific response to water deficit, we compared the so highlighted genomic differences with the DEGs found in our comparisons. We found 158 DEGs showing differences in $\mathrm{CN}$ and $336 \mathrm{SNVs}$ affecting the function of 295 DEGs. Among these, we specifically looked at genomic variants corresponding to inter cultivar DEGs related to WD responses.

We further analysed the function of those polymorphic genes and we selected putative candidate genes as detailed in [11]. Notably, we found that the higher $\mathrm{CN}$ differences affected some well-known stress-related gene families, such as ankyrin repeat proteins belonging to the RING finger family, recently described as specifically related to drought response in Arabidopsis thaliana $[15,16]$. These gene families originated by gene duplication events, therefore, in our opinion, stress conditions could have induced gene duplications and these events could create genome plasticity leading to a different ability to respond to the changing environment.

Antioxidant enzymes, metabolites, transcription regulators, and cross-talk with hormones prompted by abiotic stress conditions are crucial to ensure the right antioxidant homeostasis, achieving a positive balance between photosynthesis and respiration [17]. With this respect, among the genes showing CNVs and SNPs directly related to the expression modulation in early stress responses, we also found genes involved in photosynthesis, energetic metabolism, electron transport and ROS scavenging pathways as NADH dehydrogenases and quinone oxidoreductases.

According to the key role of ABA in the modulation of the complex hormonal network in response to $\mathrm{WD}[18,19]$, we revealed CNVs in 30 genes of phytohormone signalling and perception, most of them ABA-dependent.

As an example, higher $\mathrm{CN}$ in the gene RD22, in It, is also coupled with a significantly higher expression of these genes in cultivar It compared to AR. The Responsive to Dehydration 22 (RD22) has been recently described as a link between ABA signalling and abiotic stress responses [20] by maintaining cell integrity under stress conditions [21]. These results confirmed that ABAmediated perception and response might be the major responsible for the varietal-specific behaviour observed applying water stress.

A hypothetical scheme of the ABA-mediated mechanisms involved in responses to WD stress in cultivars $\mathrm{AR}$ and It is depicted in Fig. 3. Our results suggest that the increase of $\mathrm{ABA}$ and/or of $\mathrm{ABA}$ perception in cultivar It could be responsible for the transcriptional induction/repression of signalling genes and transcription factors, such as those belonging to AP2/AREB and MYB families. They might affect the transcriptional regulation of drought-related genes.

In contrast to the 25 ABA-responsive genes differentially expressed in It in response to WD, only two genes resulted differentially expressed in AR highlighting that ABA perception is strongly genotype-dependent. 

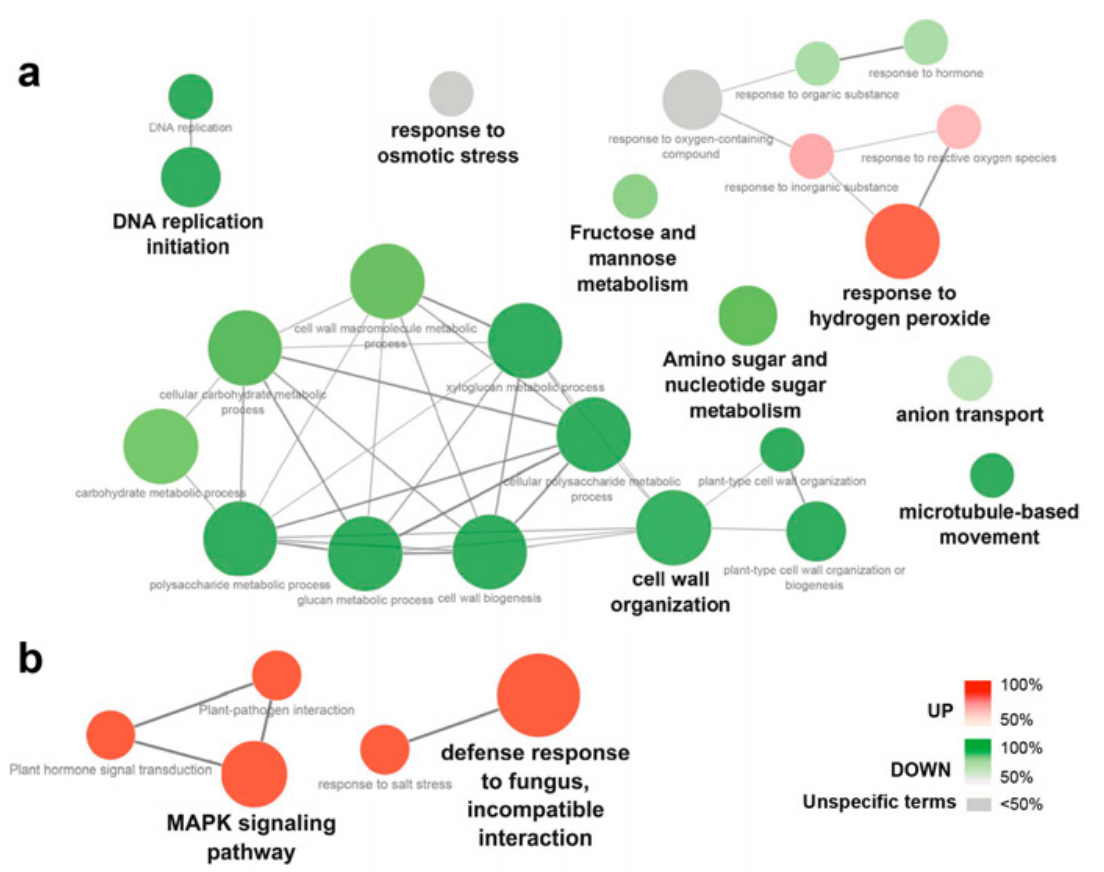

C
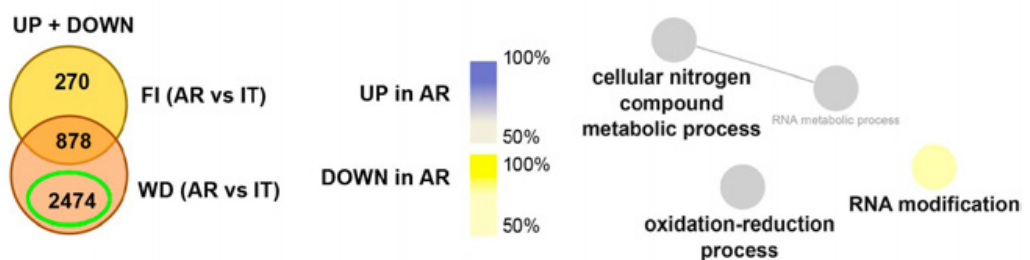

d
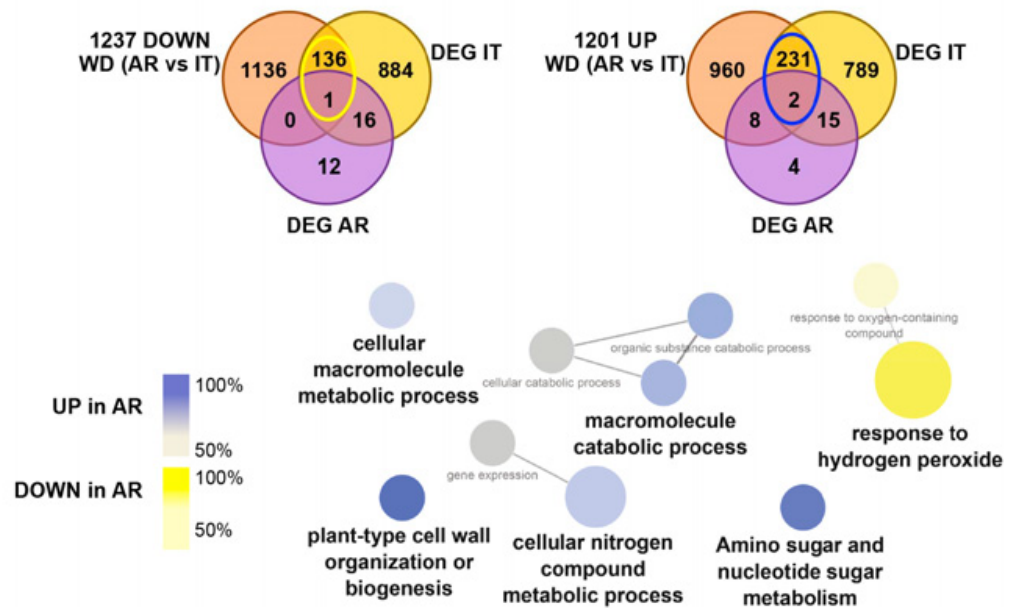

Figure 2. Main pathways involved in the response to WD in table grape cultivars Italia and Autumn Royal As modified by Catacchio et al. (2019).

(a-b) Network of genes differentially expressed under WD in cultivars It and AR, respectively. For cv. Italia only terms containing at least three genes were shown, this restriction was not applied for cv. Autumn Royal. (c-d) Network of genes differentially expressed between cvs. Italia (IT) and Autumn Royal (AR) at water deficit condition (WD) and regulated under water stress, as indicated by Venn Diagrams. Data are visualized as clusters distribution network (Cytoscape, ClueGO App). Only significant $(p<0.005)$ terms belonging to GO biological process and Kegg ontologies were shown. The node size is proportional to the term significance. The colour gradient shows the proportion of up- and down-regulated genes associated with the term. Equal proportions of both clusters are represented in gray. AR: Autumn Royal; IT: Italia; WD: water deficit; DEG: Differentially expressed genes.

\section{Conclusions}

Water deficit represents the main environmental constraint for growth in grapevine. The effects of deficit irrigation are dependent on the climatic characteristics during the growing season, soil type, and timing of application, but also on cultivar genotype and rootstock type. As consequence of these many factors, different cultivars adopt different strategies to cope with drought. However, the genetic and physiological origins of these differences are still debated $[5,9,17]$.

In order to reach new insight on these aspects we performed different trials directly on fields to test the different adaptation of grape cultivars on WD conditions also with respect to rootstock genotype. 
Table 3. CNVs and SNPs identified comparing AR and It highthroughput sequencing data. \#Genomic variants overlapping with genes belonging to both abiotic stress response and hormone signalling functional categories.

\begin{tabular}{|l|c|c|}
\hline Genomic variants & CNVs & SNVs \\
\hline Total polymorphisms & $\begin{array}{l}\text { 1249 regions } \\
\text { (1499 genes) }\end{array}$ & $\begin{array}{c}2283 \\
(1807 \text { genes })\end{array}$ \\
\hline $\begin{array}{l}\text { Overlapping with genes } \\
\text { involved in functional } \\
\text { categories of interest }\end{array}$ & $\begin{array}{c}26 \\
\text { (27 genes) }\end{array}$ & $\begin{array}{c}49 \\
\text { (41 genes) }\end{array}$ \\
\hline $\begin{array}{l}\text { Overlapping with genes } \\
\text { differentially expressed } \\
\text { between } A R \text { and } I t\end{array}$ & 159 & 336 \\
(159 genes) & $(295$ genes $)$ \\
\hline
\end{tabular}

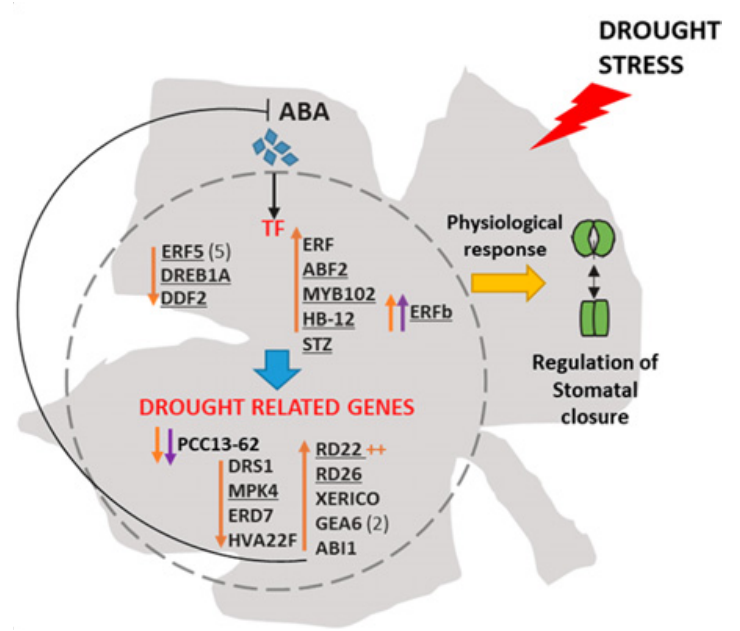

Figure 3. ABA-mediated response to drought stress in cvs. Italia vs. Autumn Royal. As modified by Catacchio et al. (2019). The ABA-responsive transcription factors (TF) belonging to AP2-EREB family: five ERF5 genes, DREB1A and DDF2 are down-regulated under WD in It, whereas, $E R F, A B F 2, M Y B 102$, the homeobox-leucin zipper protein $H B-12$, the zing-finger protein $S T Z$ are up-regulated. $E R F b$ is the only TF differentially expressed in both It and AR at WD. TF might regulate the expression of drought related for instance the down-regulation of desiccation protein PCC13-62, DRS1, MPK4, ERD7, HVA22F and the up-regulation of RD22, RD26, XERICO, GEA6, ABII. $A B I 1$ might act in a negative feedback regulatory loop of ABA. Genes whose promoters contains ABRE or ABRE-related motifs are underlined. In parentheses it is indicated the number of genes.

Our results implied that the choice of the appropriate rootstock might be crucial to improve grape quality and berry composition under limited water resource conditions and that one best choice for Mediterranean conditions is ' $140 \mathrm{Ru}$ ' which allowed in our experiments the best performance of some seedless cultivars mostly diffused in these regions.

Recently, many comparative studies have addressed the topic of water stress response in grapevine at a molecular level, using different experimental approaches $[5,9,22,23]$. With respect to previous data, we focused our attention on the early response to water deficit. Our data confirmed that WD induces modulation in genes related to response to stimuli, response to abiotic stress, ABA response, protein and carbohydrate metabolisms, nitrogen metabolism, and ROS response, thus revealing the importance of such pathways in the response to water stress. Moreover, our data strongly supports the genotypedependent response to $\mathrm{WD}$, and demonstrated a better adaptation of AR to the WD conditions. Indeed, adaptation and resilience to water stress, such as the extremely limited response in the early phase found in AR, could be considered more advantageous. In this way, the plant could activate its defence responses more gradually - only if the WD condition is prolonged - and this could avoid investing much resources and energy if not strictly necessary.

In order to understand the molecular basis of such kind of genotypic - specific response, for the first time we deeply analysed NGS data belonging to the studied cultivars and we identified candidate genes related to genotype-specific response to water deficit in grapevine.

\section{References}

[1] L.F. Casassa, R.C. Larsen, C.W. Beaver, M.S, Mireles, M. Keller, W.R. Riley, et al., J. Agric. Food Chem. 61, 6446 (2013)

[2] L.F. Casassa, M. Keller, J.F. Harbertson, Mol. 20, $7820(2015)$

[3] G. Charrier, S. Delzon, J.C. Domec, L. Zhang, CEL Delmas, I. Merlin, et al., Sci. Adv. 4 (2018)

[4] M.M. Chaves, O. Zarrouk, R. Francisco, J.M. Costa, T. Santos, A.P. Regalado, et al., 105, 661 (2010)

[5] S. Dal Santo, A. Palliotti, S. Zenoni, G.B. Tornielli, M. Fasoli, P. Paci, et al., BMC Genomics 17, 815 (2016)

[6] S. Tramontini, J. Döring, M. Vitali, A. Ferrandino, M. Stoll, C. Lovisolo, Func. Plant Biol. 41, 1119 (2014)

[7] J.C. Domec, D.M. Johnson, Tree Physiol. 32, 245 (2012)

[8] M.C. Merli, E. Magnanini, M. Gatti, F.J. Pirez, I.B. Pueyo, D.S. Intrigliolo, et al., Agric. Water Manage. 169, 106 (2016)

[9] M. Rocheta, J.L. Coito, M.J. Ramos, L. Carvalho, J.D. Becker, P. Carbonell-Bejerano, et al., BMC Plant Biol. 16, 224 (2016)

[10] R. Perniola, P. Crupi, R. Genghi, D. Antonacci (Eds.), International Society for Horticultural Science (ISHS), (Leuven, Belgium, 2016)

[11] C.R. Catacchio, F. Alagna, R. Perniola, C. Bergamini, S. Rotunno, F.M. Calabrese, et al., Sci. Rep. 9, 2809 (2019)

[12] M.F. Cardone, P. D'Addabbo, C. Alkan, C. Bergamini, C.R. Catacchio, F. Anaclerio, et al., Plant J. 88, 648 (2016)

[13] S. Koundouras, E. Hatzidimitriou, M. Karamolegkou, E. Dimopoulou, S. Kallithraka, J.T. Tsialtas, et al., J. Agric. Food Chem. 57, 7805 (2009)

[14] S.D. Castellarin, A. Pfeiffer, P. Sivilotti, M. Degan, E. Peterlunger, G. DI Gaspero 30, 1381 (2007)

[15] H. Sakamoto, O. Matsuda, K. Iba, Plant J. 56, 411 (2008)

[16] H. Sakamoto, Y. Nakagawara, S. Oguri, Int. J. Biotech. and Bioeng. 7, 1120 (2013)

[17] L.C. Carvalho, M. Silva, J.L. Coito, M.P. Rocheta, S. Amâncio, Front Plant Sci. 8, 1835 (2017)

[18] A.S. Raghavendra, V.K. Gonugunta, A. Christmann, E. Grill, Trends Plant Sci. 15, 395 (2010)

[19] T. Miyakawa, Y. Fujita, K. Yamaguchi-Shinozaki, M. Tanokura, Trends Plant Sci. 18, 259 (2013) 
[20] J.T. Matus, F. Aquea, C. Espinoza, A. Vega, E. Cavallini, S. Dal Santo, et al., PLoS One. 9, e110372 (2014)

[21] H. Wang, L. Zhou, Y. Fu, M.Y. Cheung, F.L. Wong, T.H. Phang, et al., Plant Cell Environ. 35, 1932 (2012)
[22] L.G. Deluc, D.R. Quilici, A. Decendit, J. Grimplet, M.D. Wheatley, K.A. Schlauch, et al., BMC Genomics. 10, 212 (2009)

[23] E.A. Tattersall, J. Grimplet, L. DeLuc, M.D. Wheatley, Vincent D, Osborne C, et al., Funct. Integr. Genomics. 7, 317 (2007) 